

\title{
Disentangling Intertemporal Substitution and Risk Aversion under the Expected Utility Theorem
}

Lau, Chi-Lei Oscar

Michigan State University

6 November 2008

Online at https://mpra.ub.uni-muenchen.de/11482/

MPRA Paper No. 11482, posted 10 Nov 2008 00:18 UTC 


\title{
Disentangling Intertemporal Substitution and Risk Aversion under the Expected Utility Theorem*
}

\author{
Chi-Lei Oscar Lau ${ }^{\dagger}$
}

November 6, 2008

\begin{abstract}
A disturbing feature of the conventional objective function for intertemporal decisions under uncertainty is that the agent's attitudes toward intertemporal substitution and risk aversion are entangled. This paper shows that, in contrast to common perception, the two attitudes can be completely disentangled under the expected utility theorem (EUT) by modeling each of them successively in two steps. The conventional form is nested as a special case where the functions describing the two attitudes are identical. The proposed framework requires only the standard axioms of the EUT, in addition to a regulatory assumption. It is flexible in accommodating different combinations of the two attitudes, indifferent to the timing of resolution of uncertainty, intuitive to interpret, and extendable to multiple goods.

The objective function under the proposed framework is time inconsistent according to Strotz's (1955) definition. I argue that Strotz's notion of time consistency is misguided. It is constructed based on a priori assumption that the agent should continuously forget history as time progresses, which means the agent is either chronically amnesiac or self-contradictory. To be truly consistent, the agent should have one and only one objective function, determined
\end{abstract}

*I thank Chris Ahlin, Larry Epstein, Thomas Jeitschko, Jack Meyer, Gary Solon and participants at MSU Theory Brown Bag Seminar for helpful discussions. All errors are mine.

${ }^{\dagger}$ Department of Economics, Michigan State University. Address: 110 Marshall-Adams Hall, East Lansing, MI 48824-1038. Email: lauchi@msu.edu Website: https://www.msu.edu/ lauchi/ 
at birth, throughout his entire life. As history unfolds, the agent updates his information set, but not his objective function.

KEYWORS: Intertemporal substitution; Risk aversion; Expected utility theorem; Time consistency; Equity premium puzzle

JEL Classifications: D81, D91, E21, G12

\section{Introduction}

The conventional objective function for intertemporal decisions under uncertainty takes the form of conditional expectation of sum of discounted period utilities. A disturbing feature of this setup is that the agent's attitudes toward intertemporal substitution and risk aversion are both dictated by the concavity of the period utility function, and hence tied. For instance, under the power utility form, the coefficient of relative risk aversion is constrained to be the reciprocal of the elasticity of intertemporal substitution. There is no reason to believe that this relationship should necessarily hold. Using survey data, Barsky, Juster, Kimball and Shapiro (1997) find no significant relationship, either statistically or economically, between the two attitudes. On the empirical front, there are a number of unresolved issues associated with the conventional specification, probably the best known one being the equity premium puzzle (Mehra and Prescott 1985). The puzzle concerns the inability of the conventional model to explain the large premium of returns of stocks over (nominally) riskless bonds over the long term without postulating an implausibly high degree of risk aversion. The puzzle prevails in not only the US but also other countries. Kreps and Porteus (1978), generalized by Epstein and Zin (1989), and Selden (1978) propose non-expected utility specifications that disentangle the relationship.

This paper shows that, in contrast to common perception, the expected utility theorem (EUT) is not to be blamed for the link. Actually, we can separate the two attitudes under the EUT by using a simple, axiomatic two-step approach. First, every possible consumption sequence outcome is translated into its constancy equivalent, defined as the consumption level that, if maintained throughout lifetime, will leave the agent indifferent. Next, interpreting the lotteries over consumption sequences as lotteries over constancy equivalents (scalars), we apply the EUT to the latter. The 
resulting expected utility determines the preference ordering among the lotteries. In effect, each lottery is translated into a degenerate and constant sequence (whose constant level is called its constancy-equivalent) that is equally desirable. We then only need to the compare the constancy-certainty equivalents of lotteries in order to rank them. The attitudes toward intertemporal substitution and risk aversion are encoded in the two steps independently, and therefore "cleanly" disentangled.

The conventional specification is nested as a special case where the preference under certainty is time-separable and the functions describing the two attitudes are identical. The proposed framework requires only the standard axioms of the EUT, in addition to the assumption that the agent is indifferent if the lotteries over consumption sequence are replaced by the lotteries over the corresponding constancy equivalents. The framework is flexible in accommodating different combinations of the two attitudes, indifferent to the timing of resolution of uncertainty, and intuitive to interpret. The framework can also be easily extended to accommodate multiple goods.

I will address the issue of time consistency of the objective function under the proposed framework in detail. Ever since Strotz (1955), an agent's objective function is viewed as time inconsistent if, when he arrive at a certain period, his current preference over his remaining-life consumptions (or lotteries of consumptions under uncertainty) differs from his retrospective preference (i.e. what his preference over the same remaining life would have been had he been able to foresee the future). From this perspective, the objective functions under the framework presented in this paper is time inconsistent. I argue that Strotz's notion of time inconsistency is misguided. Specifically, the "current" preference is not valid - it is constructed based on a priori assumption that the agent should continuously modify his objective function by discarding history as time progresses. But this means the agent is either amnesiac or self-contradictory. For instance, if the agent in period 0 cared about intertemporal substitution between period-0 consumption $c_{0}$ and future consumptions, then why is $c_{0}$ forgotten in period 1 ? To be truly consistent, the agent should have one and only one objective function, determined at birth, throughout his entire life. As history unfolds, the agent updates his information set, but not his objective function. The agent always cares about his full-life instead of just the remaining-life because, as 
Stroz recognizes, history can influence future decision.

Accordingly, empirical problems could arise from two sources. First, the attitudes toward intertemporal substitution and risk aversion are not properly disentangled; second, the objective function fails to cover full-life consumption sequence. In particular, the equity premium puzzle is a key empirical issue for investigation.

The rest of the paper is organized as follows. Section 2 develops the theoretical framework. Section 3 illustrates the framework with explicit functional forms, discuss its features and assumptions, and extend it to accommodiate multiple goods. Section 4 addresses the issue of time consistency. Section 5 concludes.

\section{Two-Step Approach of Disentanglement}

Consider a rational agent who is just born in period 0 , and will live until period $T$ under uncertainty. ${ }^{1}$ There is a single consumption good $c$. The conventional objective function takes the form:

$$
E_{0} \sum_{t=0}^{T} \beta^{t} \ddot{u}\left(c_{t}\right)
$$

where $\left\{c_{t}\right\}_{t=0}^{T}$ is a consumption sequence (a random vector), $\ddot{u}$ is strictly increasing, $\beta$ is the discount factor, and $E_{t}$ represents the expectation operator conditional on all information available to the agent in period $t$. The specification is simple, tractable, and intuitive, which probably accounts for its common use. Its use can be dated back to as early as Beckmann (1959), who uses the dynamic programming form. Phelps (1962) adopts (1) explicitly and explains:

This model postulates a consumer who obeys the axioms of the von Neumann-Morgenstern utility theory.

.... $[\mathrm{T}]$ he individual .... is strictly averse to risk (concavity) ... [F]or every pair of consumption histories $\left(c_{1}, \ldots, c_{N}\right)$ and $\left(c_{1}^{o}, \ldots, c_{N}^{o}\right)$ to which he is not indifferent, he will strictly prefer the certainty of the compromise history $\theta c+(1-\theta) c^{o}$ to the mixed prospect offering him the history $c$

\footnotetext{
${ }^{1}$ I will only consider the preference in period 0 (birth) in this section in order to avoid for the moment the issue of time consistency, which I will address in Section 4. $T$ can be finite or infinite.
} 
with probability $\theta$ and the history $c^{o}$ with probability $1-\theta, 0<\theta<1$. It follows trivially that $u\left(c_{n}\right)$ is a $\ldots$ strictly concave function.

Presumably, by simply adding a conditional expectation operator to the lifetime utility used in non-random models, both intertemporal substitution (variation aver$\operatorname{sion}^{2}$ ) and risk aversion are taken care of by the concavity of $\ddot{u}$ ( $u$ in the above quotation). The profession seems to have accepted that the conventional specification is a result of applying the EUT. An often cited drawback is that the preferences on intertemporal substitution and risk aversion cannot be separated because they both are dictated by $\ddot{u}$. For example, if $\ddot{u}$ takes the power utility form, then the coefficient of relative risk aversion is constrained to be the reciprocal of the elasticity of intertemporal substitution.

Another way of viewing the restriction is as follows. For convenience, consider discrete probability distributions. Under the time-separable objective function, given any lottery, the agent would be indifferent if we swap any subsequence of consumption in one state with the corresponding subsequence in any other state with equal probability. ${ }^{3}$ For instance, in a two-period setting, the agent would be indifferent between a lottery of consumption outcomes $(H, H),(L, L)$ with equal probabilities, and a lottery of $(H, L),(L, H)$ also with equal probabilities, where $H>L$. Intuitively, the second lottery have higher variation across lifetime (both outcomes have a $H$ and a $L$ ), but is less risky (will not end up with either $H$ in both periods or $L$ in both periods) than the first one. The two factors always exactly offset with each other, leaving the agent indifferent. The problem with this result is that the agent presumably make decision based on his full-life consumption sequence as a whole, instead of strands of subsequences in isolation. Whenever an objective function is time-separable, there is a cause for concern.

Before starting to disentangle the two attitudes under the EUT, it is helpful to restate what the EUT exactly says. When applied to temporal consumption decisions, the outcomes are sequences of consumptions $\left\{c_{t}\right\}_{t=0}^{T} \equiv \mathbf{c}$, rather than a single good or monetary reward as in the static case. Preferences are defined on the space of lotteries,

\footnotetext{
${ }^{2}$ I will contrast variation aversion with fluctuation aversion in Section 3.2.

${ }^{3}$ More generally, consider some probability mass being swapped even if the states do not have the same probability.
} 
each of which specifies a probability distribution of consumption sequences. The EUT claims that if the preference satisfies the axioms of completeness, transitivity, continuity and independence, then it can be represented by the expected utility form. Specifically, there exists a real valued function $w$ (the von Neumann-Morgenstern utility function) defined over the realizations of consumption sequences such that the preference can be represented by

$$
E_{0} w(\mathbf{c})
$$

The agent's attitudes toward intertemporal substitution and risk aversion are both embedded in $w$, which is unique up to positive affine transformations.

Note that the EUT is concerned only with the existence of $w$; it tells us nothing about how to postulate $w$. While the conventional specification (1) does take an expected utility form, there is no compelling reason to adopt it immediately. In fact, by doing so, we necessarily tie the two attitudes together. The tie is not a result of the EUT itself, but a consequence of unsatisfactory postulation of $w$. Indeed, conceptually none of the EUT axioms (or any combination of them) seems to necessarily lead to the tie.

\subsection{Constancy Equivalent}

Instead of attempting to postulate $w$ directly, I use a two-step approach. We already know well how to apply the EUT to scalar outcomes such as monetary amounts. When the outcomes are consumption sequences, however, we can first translate each of them into a cardinal scalar index that measures its desirability. Then we can apply the EUT to this index in the familiar fashion. The index we are going to use is called the constancy equivalent as defined below.

In the first step, consider the outcomes in deterministic environment. The preference over consumption sequences under certainty describes the agent's attitude towards intertemporal substitution and time discounting. A more variation-averse agent is more reluctant to substitute between time. A more impatient agent values future consumptions less. For each outcome, we can define its constancy equivalent as the consumption level that, if maintained throughout lifetime, will leave the agent 
indifferent.

Definition 1 (Constancy Equivalent) Given a preference under certainty represented by $U: \mathbb{R}_{+}^{T+1} \rightarrow \mathbb{R}$, for any non-random consumption sequence $\mathbf{c} \in \mathbb{R}_{+}^{T+1}$, its constancy equivalent $\bar{c} \in \mathbb{R}_{+}$is the consumption level that leaves the agent indifferent between $\mathbf{c}$ and the constant consumption sequence $\overline{\mathbf{c}} \equiv\{\bar{c}\}_{t=0}^{T}$, i.e. $U(\mathbf{c})=U(\overline{\mathbf{c}})$. Also, call $\overline{\mathbf{c}}$ the constancy equivalent sequence of $\mathbf{c}$.

No restriction on the preference under certainty is imposed as long as $\bar{c}$ exists for any c. ${ }^{4}$ Although the preference under certainty can take many different forms, the following time-separable form is often specified:

$$
U(\mathbf{c})=\sum_{t=0}^{T} \beta^{t} u\left(c_{t}\right)
$$

where the period utility function $u$ is strictly increasing and (if the agent is variationaverse) strictly concave, and the discount factor is $\beta$. The constancy equivalent of $\mathbf{c}$ is given by $\bar{c}$ such that

$$
\sum_{t=0}^{T} \beta^{t} u(\bar{c})=\sum_{t=0}^{T} \beta^{t} u\left(c_{t}\right)
$$

or

$$
\bar{c}=u^{-1}\left(\frac{1-\beta}{1-\beta^{T+1}} \sum_{t=0}^{T} \beta^{t} u\left(c_{t}\right)\right)
$$

\subsection{Constancy Equivalence EUT}

In the second step we move to the stochastic environment. In doing so, we need to assume that constancy equivalence carries over to the stochastic environment. Specifically, we make the constancy indifference assumption: given any lottery of consumption sequences, the agent is indifferent if the consumption sequence $\mathbf{c}$ in each outcome is replaced by its constancy equivalent sequence $\overline{\mathbf{c}}$. By virtue of this constancy indifference assumption, we can convert the problem of choosing between lotteries of consumption sequences into one of choosing between lotteries of constancy equivalents. We can therefore apply the EUT with the constancy equivalents treated

\footnotetext{
${ }^{4} \mathrm{~A}$ preference that fails this requirement is the lexicographic preference.
} 
as outcomes. By the EUT, there exists a function $v$ such that the preference can be represented by $E_{0} v(\bar{c})$. Formally, ${ }^{5}$

Lemma 2 (Constancy Equivalence EUT) Suppose that the constancy indifference assumption hold. If a preference on the space of lotteries of consumption sequences satisfies the completeness, transitivity, continuity and independence axioms, then there exists a constancy equivalence von Neumann-Morgenstern utility function $v: \mathbb{R}_{+} \rightarrow \mathbb{R}$ such that the preference in period 0 can be represented by the objective function

$$
E_{0} v(\bar{c})
$$

where $\bar{c}$ denotes the constancy equivalent.

The theorem is a specialization of the orginal dynamic EUT (2), where $w$ is defined jointly by $U$ and $v$. The theorem and $v$ are so named to distinguish them from the original dynamic EUT and $w$. The function $v$ is defined on constancy equivalents, whereas $w$ on consumption sequences. If all lotteries are degenerate, then (5) represents exactly the same preference as the preference under certainty. Notice that $v$, with certainty equivalent as the argument, describes risk aversion toward fulllife consumption as a whole. Contrast this with the conventional specification, where $\ddot{u}$ concerns risk aversion toward single period lotteries.

If the preference under certainty takes the time-separable form (3), then $w$ is defined jointly by $u, v$ and $\beta$. Any outcome of consumption sequence is translated to its constancy equivalent by $u$ and $\beta$, which then contribute to the objective function by mapping through $v$. The agent's attitude towards intertemporal substitution and risk are represented by $u$ and $v$ respectively, and hence they are cleanly separated. In the special case where $u=v,(5)$ is equivalent to the familiar conventional specification (just substitute (4) into (5)). Note that even when the preference under certainty is time-separable, the objective function is not in general.

Corresponding to the notions of certainty equivalent and risk premium in the static setting, we can define their counterparts in the dynamic environment:

\footnotetext{
${ }^{5}$ Strictly speaking, the theorem only requires the EUT axioms to hold for the lotteries on certainty equivalent sequences, rather than for the lotteries on all consumption sequences. But the more general assumption appears to be a more natural one to make. In addition, it guarantees the existence of constancy equivalents.
} 
Definition 3 (Certainty-constancy Equivalent) Given a lottery on consumption sequences, define its certainty-constancy equivalent $\widehat{c}$ as the non-random scalar such that the agent is indifferent between the lottery and a degenerate constant consumption sequence $\widehat{\mathbf{c}} \equiv\{\widehat{c}\}_{t=0}^{T}$.

Under the general form of dynamic EUT (2), $w(\widehat{\mathbf{c}})=E_{0} w(\mathbf{c})$. Under the constancy equivalence EUT (5), $\widehat{c}$ is simply the certainty equivalent of the random $\bar{c}$ : $v(\widehat{c})=E_{0} v(\bar{c})$.

The scalar $\widehat{c}$ can be viewed as a lottery's summary score that incorporates all aspects of the agent's preference under temporal stochastic environment. Hence the agent chooses whichever lottery that yields the highest $\widehat{c}$.

\section{Definition 4 (Variation Premium, Risk Premium and Risk-variation Premium)}

Given a lottery on consumption sequences, the variation premium $\mathbf{p}^{v} \equiv E_{0}(\mathbf{c})-$ $E_{0}(\overline{\mathbf{c}})$, the risk premium $\mathbf{p}^{r} \equiv E_{0}(\overline{\mathbf{c}})-\widehat{\mathbf{c}}$, and the risk-variation premium $\mathbf{p}^{r v} \equiv E_{0}(\mathbf{c})-\widehat{\mathbf{c}}$.

Therefore, $\mathbf{p}^{v}$ is the sequence of period-by-period differences between the expected value of consumption under the lottery and that of the variation-free (but uncertain) $\overline{\mathbf{c}}$. Intuitively, $\mathbf{p}^{v}$ is the expected value of consumption that the agent is willing to sacrifice in order to get rid of variations within any outcome of consumption sequence. Similarly, $\mathbf{p}^{r}$ is the expected value of consumption that the agent is willing to sacrifice in order to further get rid of uncertainty. $\mathbf{p}^{r v}$ is simply the sum of the two: the expected value to be sacrificed in order to get rid of both variation and uncertainty.

Figure 1 illustrates the constancy-equivalence EUT for the simple case of two periods ( 0 and 1$)$ and a lottery of two outcomes $(A$ and $B)$ with equal probabilities. The bottom panel shows the indifference curves $\left(I C_{A}\right.$ and $\left.I C_{B}\right)$ associated with the two outcomes under certainty. Because of discounting of period 1 utility, the indifference curves are not symmetric around the $45^{\circ}$ line. The constancy equivalents $\bar{c}_{A}$ and $\bar{c}_{B}$ are given by the intersections between the indifference curves and the $45^{\circ}$ line. The constancy equivalents are then mapped to $v\left(\bar{c}_{A}\right)$ and $v\left(\bar{c}_{B}\right)$ in the top panel, from where we can find $E_{0} v(\bar{c})$ and thus the certainty-constancy equivalent $\widehat{c}$. Any lottery can be mapped to its certainty-constancy equivalent in a similar fashion. This is how 
the lotteries are ranked. The preference under certainty is shaped by $U$ in the bottom panel, whereas the risk attitude is dictated by $v$ in the top panel.

The agent is indifferent between the lottery $(A, B)$ and the lottery $(\bar{A}, \bar{B})$ by virtue of the constancy indifference assumption, and also the degenerate-and-constant sequence denoted by $\hat{C}$. $M$ is the midpoint of $A$ and $B$, whereas $\bar{M}$ is the midpoint of $\bar{A}$ and $\bar{B}$. Then $\mathbf{p}^{v}$ is the vector pointing from $\bar{M}$ to $M, \mathbf{p}^{r}$ from $\hat{C}$ to $\bar{M}$, and $\mathbf{p}^{r v}$ from $\hat{C}$ to $M$.

We can also see from Figure 1 why the conventional specification is recovered when $u=v$ (assuming the time-separable form for the preference under certainty). If $u$ and $v$ coincides, then we can replace $v$ by $u$ in the top panel. $E_{0} v(\bar{c})$ becomes

$E_{0} u(\bar{c})=\frac{1}{2} u\left(\bar{c}_{A}\right)+\frac{1}{2} u\left(\bar{c}_{B}\right)$, which equals $\frac{1}{2}\left[u\left(c_{A 0}\right)+\beta u\left(c_{A 1}\right)\right]+\frac{1}{2}\left[u\left(c_{B 0}\right)+\beta u\left(c_{B 1}\right)\right]$ by definition of constancy equivalent. Thus the conventional specification is recovered.

\section{Discussion}

This section illustrates the constancy equivalence EUT framework with explicit functional forms, discuss its features and assumptions, and extend it to accommodiate multiple goods.

\subsection{Explicit Functional Forms}

The functional forms for $U$ and $v$ are flexible. For instance, an agent can have Leontief preference for $U$ and linear preference for $v$. However, for illustration, I assume the time-separable form for the preference under certainty, and specify the functional forms of $u$ and $v$ as follows. Suppose $u$ takes the constant elasticity of intertemporal substitution (CEIS) form:

$$
u(c)= \begin{cases}c^{1-\sigma} /(1-\sigma) & \text { if } \quad \sigma \geq 0, \sigma \neq 1 \\ \log (c) & \text { if } \quad \sigma=1\end{cases}
$$

where $1 / \sigma$ is the elasticity of intertemporal substitution for consumption sequences under certainty. Meanwhile, $v$ takes the form of constant relative risk aversion (CRRA): 


$$
v(\bar{c})= \begin{cases}\bar{c}^{1-\rho} /(1-\rho) & \text { if } \quad \rho \geq 0, \rho \neq 1 \\ \log (\bar{c}) & \text { if } \quad \rho=1\end{cases}
$$

where $\rho$ is the coefficient of relative risk aversion for lotteries of constancy equivalents. The parameter $\rho$ should be interpreted as measuring risk aversion toward lifetime consumptions.

The attitudes toward intertemporal substitution and risk aversion are determined independently by $\sigma$ and $\rho$ respectively. Suppose $\sigma, \rho \neq 1$, then

$$
\bar{c}=\left[\frac{1-\beta}{1-\beta^{T+1}}\left(\sum_{t=0}^{T} \beta^{t} c_{t}^{1-\sigma}\right)\right]^{\frac{1}{1-\sigma}}
$$

the objective function $(5)$ becomes (dropping the positive term $\left.\left(\frac{1-\beta}{1-\beta^{T+1}}\right)^{\frac{1-\rho}{1-\sigma}}\right)^{6}$

$$
E_{0}\left[\frac{1}{1-\rho}\left(\sum_{t=0}^{T} \beta^{t} c_{t}^{1-\sigma}\right)^{\frac{1-\rho}{1-\sigma}}\right]
$$

The familiar conventional specification for power utility form is recovered when $\sigma=\rho$. To the extent that $\sigma$ and $\rho$ are different, using the conventional objective function would cause significant departure from observed data, and thus lead to empirical issues. Also notice that even though the preference under certainty is time-separable, the objective function is not, unless $\sigma=\rho$.

If the agent is variation-neutral $(\sigma=0)$, then $(6)$ becomes

$$
E_{0}\left[\frac{1}{1-\rho}\left(\sum_{t=0}^{T} \beta^{t} c_{t}\right)^{1-\rho}\right]
$$

In this case, all that the agent cares ex post is the discounted sum of consumptions $\sum_{t=0}^{T} \beta^{t} c_{t}$. Ex ante, the agent maximizes the conditional expectation of $v$ of the discounted sum in accordance with the EUT.

$$
{ }^{6} \text { Also, } \hat{c}=\left\{E_{0}\left\{\left[\frac{1-\beta}{1-\beta^{T+1}}\left(\sum_{t=0}^{T} \beta^{t} c_{t}^{1-\sigma}\right)\right]^{\frac{1-\rho}{1-\sigma}}\right\}\right\}^{\frac{1}{1-\rho}}
$$


On the other hand, if the agent is risk-neutral $(\rho=0)$, then $(6)$ becomes

$$
E_{0}\left[\left(\sum_{t=0}^{T} \beta^{t} c_{t}^{1-\sigma}\right)^{\frac{1}{1-\sigma}}\right]
$$

One may wonder why the objective function does not reduce to the conventional form even when the agent is risk-neutral. The answer is that the agent cares about the fulllife consumption sequence as a whole. If the objective function were time-separable, then we are ignoring the variations of consumptions within each outcome; the agent would be indifferent if we swap a subsequence of consumption in one state with the corresponding subsequence in any other state with equal probability. But this result contradicts with the premise that the agent is variation-averse.

\subsection{Features and Assumptions}

The constancy indifference assumption postulates that the agent facing a lottery is indifferent if each outcome (a full-life consumption sequence) is now replaced by the corresponding constancy equivalent sequence. Suppose the lottery were drawn right now and the outcome were revealed completely to the agent, then by definition of constancy equivalent the agent is indifferent between the realized consumption sequence and the constancy equivalent sequence. However, in reality the complete outcome is not known until the agent dies. Hence the constancy indifference assumption can be understood as indifference to early resolution of uncertainty. ${ }^{7}$

If the agent does not care whether the uncertainty is resolved early, then the constancy indifference assumption is satisfied. Together with the other axioms, the constancy equivalence EUT says that the preference can be represented by (5), which itself implies indifference to early resolution. ${ }^{8}$

Therefore the constancy-equivalence EUT is logically coherent with respect to the constancy indifference assumption: If we assume indifference to early resolution,

\footnotetext{
${ }^{7}$ Indifference to early resolution can be understood intuitively as follow. Suppose the agent is informed that the entire consumption sequence will be revealed later today, the agent is neither more nor less happy before the revelation.

8 (5) is already conditioned on currently available information; taking expectation conditional on currently available information again makes no difference.
} 
then we get a preference representation that does conform with this property. So indifference to early resolution is a feature of the EUT framework.

Sure enough, the EUT itself is not without controversy regardless of the constancy indifference assumption. ${ }^{9}$ The independence axiom in particular is subject to much scrutiny. This paper makes no attempt to investigate this topic. However, the framework developed here refutes the line of arguments against the EUT that is based on the perceived link between intertemporal substitution and risk aversion.

Another assumption made in the specification is that $T$ (if finite) is known. While this assumption by itself is unrealistic, the potential problem could be mitigated by interpreting $\beta$ as not just reflecting impatience, but also uncertainty of when the life ends.

Next, it would be worthwhile to distinguish between variation aversion and fluctuation aversion. For the preference under certainty, the time-separable form addresses only impatience and variation aversion. In the absence of discounting, the agent is indifferent between any permutation of a consumption sequence. For instance, the agent is indifferent if a consumption sequence consisting of $L$ for the first half and $H>L$ for the second is replaced by a sequence in which $H$ and $L$ alternates. The agent cares about variations in quantities, but not the period-by-period fluctuations per se.

In contrast, under the habit formation models (for example, Constantinides, 1990; Campbell and Cochrane, 1995), the agent does care about how the consumption sequence is ordered even in the absence of discounting. In principle, the constancy equivalence EUT framework can allow habit formation and order dependence. There is no restriction on the preference under certainty as long as constancy equivalent exists. Also notice that the attitudes toward variations and fluctuations are sensitive to the choice of length of period in discrete models. For instance, a sequence alternating between $H$ and $L$ will be completely flat if we double the length of time in each period. Variations and fluctuations within a period are ignored.

Finally, in the two-step approach we eliminate variation in the first step and risk in the second. One may wonder whether we could reverse the procedure, i.e. to eliminate risk first and then variation next. A natural way to proceed would be as

\footnotetext{
${ }^{9}$ See Starmer (2000) for a survey of non-expected utility theories.
} 
follows. Given a lottery, find the certainty equivalent consumption for each period separately. (Note that the risk attitude could vary across periods.) We thus arrive at a degenerate but non-constant consumption sequence, which we then "smoothen" into a degeneate constant sequence according to the agents attitude toward variation aversion. Unfortuantely, this approach is not only more complicated, but also wrong. In reducing a lottery of different outcomes into a sequence of certainty equivalents period-by-period, we are treating each period as isolated objects and therefore lose the intertemporal relationship within each outcome. The mistake is similar to the one made in the conventional objective function.

\subsection{Extension to Multiple Goods}

We can easily generalize the constancy equivalent EUT framework to accommodate multiple goods. A leading application is the labor supply decision where the agent chooses leisure as well as income consumptions. Another example is macroeconomic models that distinguish between durable and non-durable goods. In general, suppose there are $N$ goods and the preference under certainty is represented by $U: \mathbb{R}_{+}^{(T+1) \times N} \rightarrow \mathbb{R}$. For any non-random sequence of consumption bundles $\mathbf{x}=\left\{x_{1 t}, x_{2 t}, \ldots, x_{N t}\right\}_{t=0}^{T}$ where $x_{i t}$ denotes the consumption of good $i$ in period $t$, define its constancy equivalent $\bar{x}$ by the following relationship:

$$
U(\mathbf{x})=U(\overline{\mathbf{x}})
$$

where $\overline{\mathbf{x}}=\{\bar{x}, \bar{x}, \ldots, \bar{x}\}_{t=0}^{T}$. In other words, the agent is indifferent if the consumption level of each good in each period becomes $\bar{x}$ invariably. As long as the constancy indifference assumption together with the axioms of the EUT hold, we can rank the lotteries over the sequences of multiples goods by $E_{0} v(\bar{x})$, or equivalently by the certainty constancy equivalent $\hat{x}$ defined by $v(\hat{x})=E_{0} v(\bar{x})$.

In picking the units of measurements for the different goods, it is advisable to make "sensible" choices such that the equality of number of units of all goods would seem plausible. For example, if the typical worker earn about $\$ 40,000$ by working for about 2,000 hours (i.e. 6,760 leisure hours) per year. Then we could choose to measure leisure in hours and income consumption in six dollars. The advantage of 
picking commensurate units of measurement is twofold. First, it helps avoid reaching constraints such as maximum number of hours available for leisure. Second, typically the utility function is intended to describe trade-off relationship between goods for the regions of realistic consumption levels.

There remains the question of specifying $U$. As an example, a typical functional form involving two goods - non-durables $(c)$ and durables $(d)$ - is

$$
U(\mathbf{c}, \mathbf{d})=\sum_{t=0}^{T} \beta^{t}\left[\gamma \log c_{t}+(1-\gamma) \log d_{t}\right]
$$

However, in light of the theme of this paper, one may be concerned with the entangling of attitutdes toward intertemporal substitution (across time) and intratemporal substitution (across contemporaneous goods). According to the above preference, in the absence of discounting, it does not matter which $c$ and $d$ is paired in each period, as long as the entire (unordered) set $\left\{c_{t}, d_{t}\right\}_{t=0}^{T}$ remains the same. For instance, in a two-period setting, a sequence of $\left\{\left(c_{H}, d_{H}\right),\left(c_{L}, d_{L}\right)\right\}$ is just as desirable as $\left\{\left(c_{H}, d_{L}\right),\left(c_{L}, d_{H}\right)\right\}$. The intratemporal substitution / complementarity relationship between $c$ and $d$ is mixed with intertemporal relationship.

In light of the two-step approach, a three-step approach in the similar spirit can be applied here to disentangle the intertemporal, intratemporal and risk attitudes altogether. First, for each outcome, convert the consumption bundle $\left\{x_{1 t}, x_{2 t}, \ldots, x_{N t}\right\}$ in each period $t$ into its period-specific constancy equivalent $\bar{x}_{t}$ given by $p\left(x_{1 t}, x_{2 t}, \ldots, x_{N t}\right)=$ $p\left(\bar{x}_{t}, \bar{x}_{t}, \ldots, \bar{x}_{t}\right)$, where $p$ dictates the intratemporal attitude. (Here I assume that ther intratemporal attitude is the same across time, so that we need only one $p$.) Next, make the period-specific constancy indifference assumption: that the agent is indifferent if the consumption bundle in each period of a sequence is replaced by its period-specific constancy equivalent bundle. In other words, $\mathbf{x}=\left\{x_{1 t}, x_{2 t}, \ldots, x_{N t}\right\}_{t=0}^{T}$ is as preferred as $\left\{\bar{x}_{t}, \bar{x}_{t}, \ldots, \bar{x}_{t}\right\}_{t=0}^{T}$. We can then translate the whole consumption sequence $\mathbf{x}$ into its constancy equivalent $\bar{x}$ such that $q\left(\bar{x}_{0}, \bar{x}_{1}, \ldots, \bar{x}_{T}\right)=q(\bar{x}, \bar{x}, \ldots, \bar{x})$, where $q$ represents the intertemporal attitude as well as discounting. Finally, in moving to the stochastic environment, make the constancy indifference assumption and apply the constancy equivalence EUT.

In essence, $U$ is defined jointly by $p$ and $q$. The three steps take care of intratem- 
poral substitution, intertemporal substitution, and risk aversion successively. As an illustration, assume constant elasticities $1 / \theta$ and $1 / \sigma$ for the two substitutions respectively, and constant relative risk aversion of coefficient $\rho$ for lotteries on constancy equivalents, i.e. ${ }^{10}$

$$
\begin{aligned}
p\left(x_{1 t}, x_{2 t}, \ldots, x_{N t}\right) & =\sum_{i=1}^{N} \frac{x_{i t}^{1-\theta}}{1-\theta} \\
q\left(\bar{x}_{0}, \bar{x}_{1}, \ldots, \bar{x}_{T}\right) & =\sum_{t=0}^{T} \beta^{t} \frac{\bar{x}_{t}^{1-\sigma}}{1-\sigma} \\
v(\bar{x}) & =\frac{\bar{x}^{1-\rho}}{1-\rho}
\end{aligned}
$$

then it is straightforward to show that the objective function takes the form of (with some positive constant terms dropped) $)^{11}$

$$
E_{0}\left\{\frac{1}{1-\rho}\left[\sum_{t=0}^{T} \beta^{t}\left(\sum_{i=1}^{N} x_{i t}^{1-\theta}\right)^{\frac{1-\sigma}{1-\theta}}\right]^{\frac{1-\rho}{1-\sigma}}\right\}
$$

Finally, I leave the issues of (i) whether it is important to disentangle the intratemporal and intertemporal attitudes, and (ii) whether it is necessary to define an even more nuanced intratemporal relationship between various goods (rather than relying on a single parameter such as $\theta$ above) as empirical questions.

\section{Time Consistency}

Strotz (1955) establishes the notion of time consistency, which has been adopted by the profession ever since:

An individual is imagined to choose a plan of consumption for a future period of time so as to maximize the utility of the plan as evaluated at

\footnotetext{
${ }^{10}$ Each of the functions take the $\log$ form when the relevant parameter $(\theta, \sigma, \rho)$ is 1 .

${ }^{11}$ Also, the certainty-constancy equivalent $\hat{x}=\left\{E_{0}\left\{\left[\frac{1-\beta}{1-\beta^{T+1}} \sum_{t=0}^{T} \beta^{t}\left(\frac{1}{N} \sum_{i=1}^{N} x_{i t}^{1-\theta}\right)^{\frac{1-\sigma}{1-\theta}}\right]^{\frac{1-\rho}{1-\sigma}}\right\}\right\}^{\frac{1}{1-\rho}}$
} 
the present moment.... Our problem arises when we ask: If he is free to reconsider his plan at later dates, will he abide by it or disobey it - even though his original expectations of future desires and means of consumption are verified? Our answer is that the optimal plan of the present moment is generally one which will not be obeyed, or that the individual's future behavior will be inconsistent with his optimal plan.

According Strotz's definition, the objective function under the constancy equivalence EUT framework is time inconsistent in general. I will argue that this definition of time consistency is misguided. Strotz's presumption that the agent should maximize the utility of the plan "as evaluated at the present moment" means that the agent keep modifying his "current" objective function by discarding history as time progress. Actually, the creation of the "current" objective function implies the agent is either amnesiac or self-contradictory. To be truly consistent, the agent should have one and only one objective function, determined at birth, throughout his lifetime. As history unfolds, the agent updates his information set, but not his objective function. The agent always cares about his full-life instead of just the remaining-life because, as Stroz and other researchers recognize, history can influence future decision.

In this section, I first motivate the issue by laying out Epstein and Zin's (1989) concern on time inconsistency in the context of the constancy equivalence EUT framework. I then argue that the concern is misguided. With this perspective, I contend that it is the class of objective functions defined on remaining-life, which includes all recursively defined objective functions, that is indeed incoherent.

\subsection{The Concern for Time Inconsistency}

The constancy equivalence EUT objective function is reminiscent of Kihlstrom and Mirman's (1974) approach in that it maps the random variable ( $\bar{c}$ in this case) through the function $v$ before taking expectation in order to account for risk aversion. Epstein and Zin (1989, pp. 950-952) caution that when applied to the temporal environment, the Kihlstrom-Mirman approach would result in time inconsistency. To see the concern, consider an agent who arrives at period $R$. According to Strotz, his updated objective function should now be 


$$
E\left[v_{R}\left(\bar{c}_{R}\right) \mid I_{R}\right]
$$

where $I_{R}$ is the set of all information that the agent possesses in period $R .^{12}$

On the other hand, suppose that the agent's consumption history is $\left(\dot{c}_{0}, \dot{c}_{1}, \ldots, \dot{c}_{R-1}\right)$ and he contemplates his decision in period 0 retrospectively. In period 0 , had he been able to tell the future (i.e. know $I_{R}$ completely, which includes among other things his information in period 0 and his consumption history $\left(\dot{c}_{0}, \dot{c}_{1}, \ldots, \dot{c}_{R-1}\right)$ to be realized), his objective function in period 0 would have been

$$
E\left[v_{0}\left(\bar{c}_{0}\right) \mid I_{R}\right]
$$

where $\bar{c}_{0}$ is calculated partly based on deterministic values $\left(\dot{c}_{0}, \dot{c}_{1}, \ldots, \dot{c}_{R-1}\right)$.

The concern is that since $v$ is not linear in general, the two objective functions could result in different preferences. To see this more clearly, consider the CEISCRRA form. The two objective functions become ${ }^{13}$

$$
E\left[\frac{1}{1-\rho}\left(\sum_{t=R}^{T} \beta^{t-R} c_{t}^{1-\sigma}\right)^{\frac{1-\rho}{1-\sigma}} \mid I_{R}\right]
$$

for the "current" problem and

$$
E\left[\frac{1}{1-\rho}\left(\sum_{s=0}^{R-1} \beta^{s} \dot{c}_{s}^{1-\sigma}+\sum_{t=R}^{T} \beta^{t} c_{t}^{1-\sigma}\right)^{\frac{1-\rho}{1-\sigma}} \mid I_{R}\right]
$$

for the retrospective problem. The two objective functions would represent different preferences for the lottery on consumption sequences $\left(c_{R}, c_{R+1}, \ldots, c_{T}\right)$, hence resulting in time inconsistency. The agent may prefer one lottery over another today, but he would have chosen the opposite yesterday (even with foresight of what would happen today). ${ }^{14}$ Moreover, the result is not restricted to the time-separable form of

\footnotetext{
${ }^{12}$ The function $v$ is subscripted with the time period to allow for finite life: The risk aversion toward remaining full-life consumption can depend on how long the remaining life is. Similarly, the formula for $\bar{c}$ depends on the length of the remaining life.

${ }^{13}$ Assume for simplicity that $\sigma$ and $\rho$ do not change with the length of remaining life.

${ }^{14}$ The issue can be analyzed equivalently by a prospective rather than retrospective thought ex-
} 
preference under certainty.

\subsection{Full-life versus Remaining-life Objective Functions}

Having laid out the issue, now I argue that it is a misguided concern. While the retrospective objective function is valid, the current one is not. As the profession recognize, the objective function can be history-dependent in general. This brings us back to the recurring message of this paper on intertemporal choices: Agents care about full-life consumption sequence beginning with period 0 (when he was born) as a whole, not just the remaining life, or any other subsequence. History is history, but history still matters because the agent cares about his full-life consumption sequence as a whole. For instance, if past consumptions have been low, the agent would be less averse to lotteries that entail relatively low level but also less risky future consumption, as compared to another situation where past consumptions have been high. This is because low future consumptions would mean high full-life variations for the latter situation but relatively low variations for the former. ${ }^{15}$ Without forgetting history, the objective function is always

$$
E\left[\frac{1}{1-\rho}\left(\sum_{t=0}^{T} \beta^{t} c_{t}^{1-\sigma}\right)^{\frac{1-\rho}{1-\sigma}} \mid I_{D}\right]
$$

throughout the lifetime. $I_{D}$ is the set of all information available to the agent, which contains, among other information, consumption history up to period $D-1$. $^{16}$

It should be clear from (7) that the only source of change for the agent's preference is due to changes in the prevailing information set $I_{D}$. The agent periodically update his preference and plan with new information. But a consistent agent never update the objective function itself. Even equipped with the current (period $D$ ) information set, his objective function still takes the form of (7).

Except for rare situations, the agent's information set has to change with time, for

periment: Suppose now is period 0, and the agent look forward to his decision in period $R$ given the consumption history to be realized and the future information set.

${ }^{15}$ The general idea of history dependence should not be confused with the more specific notion of habit formation (see Section 3.2).

${ }^{16}$ I do not "dot" the realized consumptions for the sake of uniformity of (7) across time; the dotting is implied by $I_{D}$. 
at least consumption history and some other random events relevant to the agent's objective function must be resolved as history unfolds. The "rare" situation occurs when some consumption subsequence is predetermined, and he is isolated from new information. Perhaps the example closest to this situation is a prisoner serving in isolation. $^{17}$

Given the profession's recognition of history dependence of preference, it is unclear as to why another "current" objective function that disregards history would be artificially created. Presumably the logic is that since history is already realized, it should play no role in a forward-looking agent's objective function. But this reasoning exactly contradicts with the notion of history dependence of preference. For instance, if the agent in period 0 cared about intertemporal substitution between period-0 consumption $c_{0}$ and future consumptions, then why is $c_{0}$ forgotten in period 1? Conversely, if the agent in period 0 foresaw that he will disregard $c_{0}$ in the next period, why would he care about $c_{0}$ now? Under the "current" objective function, the agent does not only forget what happened before, he even forget that he has existed before. Equivalently, he is assumed to be reborn at the beginning of every period.

We can perhaps step back and rethink about the issue at a conceptual level. The constancy equivalence EUT framework is constructed axiomatically. Just as the EUT axioms does not seem to necessarily lead to entangling of intertemporal substitution and risk aversion, it does not appear that any of the axioms or the constancy indifference assumption (or any combination of them) should result in time inconsistency. And they do not.

In Section 2, the objective function is stated for period 0 only, which is the period of birth. Now we are ready to define the general objective function that prevails throughout the full-life. Suppose the agent is born in period 0 and will die in period $T$, then his one and only one objective function is always

$$
E\left[v(\bar{c}) \mid I_{D}\right]
$$

where $I_{D}$ is the set of all information available to the agent in the current period $D$

\footnotetext{
${ }^{17}$ If he is not jailed in isolation, then his information set will still change (e.g. through watching news or meeting his visiting wife). Although he has no economic decision to make during his jail term, he does have an objective function and hence preference.
} 
(between 0 and $T$ ); and $\bar{c}$ is calculated on full-life basis, even though some consumptions may have already been determined:

$$
U(\overline{\mathbf{c}})=U(\mathbf{c})
$$

where $\overline{\mathbf{c}} \equiv\{\bar{c}\}_{t=0}^{T}$ and $\mathbf{c} \equiv\{c\}_{t=0}^{T}$.

Epstein and Zin raise another concern on the Kihlstrom-Mirman type of objective functions: If $\beta<1$, then the dependence of preference on past consumption is greater as the past becomes more distant. Now under the principle that the agent care about full-life utility as a whole, it does not seem to be a problem. Actually, the very fact that $\beta<1$ exactly means earlier consumption are given heavier weights. ${ }^{18}$

\subsection{Amnesia of Remaining-life Objective Functions}

The remaining-life perspective sees the constancy equivalence EUT preference as inconsistent only because the former imposes a priori assumption that agents keep forgetting history, or alternatively, that agents are reborn in each period. On the other hand, recursively defined objective functions by its very own nature automatically guarantee fulfillment of Strotz's notion of time consistency because the preference in each period is made to be history independent. From the full-life perspective, it is actually the remaining-life setting, which include all recursively defined objective functions, that is incoherent. For example, consider Epstein-Zin's recursively defined objective function for period $t$ with explicit functional form:

$$
U_{t}=\left[(1-\beta) c_{t}^{\gamma}+\beta\left(E_{t} U_{t+1}^{\alpha}\right)^{\gamma / \alpha}\right]^{1 / \gamma}
$$

where $\gamma$ and $\alpha$ are parameters dictating the attitudes toward intertemporal substitution and risk aversion respectively. In period $0, c_{0}$ enters the objective function $U_{0}$. In period 1 , however, $c_{0}$ appears nowhere in the objective function $U_{1}$. If the agent cared about intertemporal substitution between $c_{0}$ and future utility in period 0 , why

\footnotetext{
${ }^{18}$ Moreover, the "discount" factor does not have to be less than one, even with the uncertainty of $T$ taken into consideration. If the agent is mortal, discounting is not necessary for obtaining finite lifetime utility. There are also evidences for a negative time-discount rate. See, for example, Barsky et. al. (1997) and Loewenstein and Prelec (1992).
} 
is $c_{0}$ forgotten in period 1 ?

In fact, all objective functions that are defined only on the remaining-life, including all recursive objective function, suffer from chronic amnesia in principle. However, in some special cases, the ever changing remaining-life objective function happens to be equivalent to the true full-life objective function. An obvious example is the conventional specification. Strictly speaking, the conventional objective function in say, period 1 should be

$$
E_{1}\left[u\left(\dot{c}_{0}\right)+\sum_{t=1}^{T} \beta^{t} u\left(c_{t}\right)\right]
$$

But due to time separability, this "happens" to be equivalent to the familiar form:

$$
E_{1}\left[\sum_{t=1}^{T} \beta^{t} u\left(c_{t}\right)\right]
$$

The conventional specification is a convenient way to avoid the amnesia problem. Unfortunately, it achieves that only at the expense of entangling intertemporal substitution and risk aversion. The constancy equivalence EUT objective function (8) is not recursive, but this is inevitable because agents care about full-life consumption as a whole.

\section{Conclusion}

This paper proposes a two-step approach to cleanly disentangle an agent's attitudes toward intertemporal substitution and risk aversion. The approach is based on the concept of constancy equivalent. It is axiomatically constructed, flexible, indifferent to the timing of resolution of uncertainty, and intuitive to interpret. It can also be extended to accommodate multiple goods. Another contribution of the paper is to point out that to be consistent, an agent should have one and only one objective function, determined at birth, that is defined on full-life. A recurring message is that the agent cares about the full-life consumption sequence as a whole. Objective functions defined on remaining-life only, including recursive objective functions, are amnesiac because they keep forgetting history. 
Given the constancy equivalence EUT framework, the obvious step next is to put the theory to test. The framework can be evaluated with respect to (1) its disentangling performance, and (2) its assertion of full-life objective function. The financial markets should serve as the ideal testing grounds. In particular, the equity premium puzzle would be a key empirical issue to investigate.

\section{References}

Barsky, R. B., F. T. Juster, M. S. Kimball and M. D. Shapiro (1997): "Preference Parameters and Behavioral Heterogeneity: An Experimental Approach in the Health and Retirement Study," Quarterly Journal of Economics, May 1997, 112(2), 537-579.

Beckmann, M. J. (1959): "A Dynamic Programming Model of the Consumption Function," Cowles Foundation Discussion Paper No. 68, March 1959.

Campbell, J. Y. and J. H. Cochrane (1999), "By force of habit: A consumptionbased explanation of aggregate stock market behavior," Journal of Political Economy 107, 205-251.

Constantinides G. M. (1990): "Habit Formation: A Resolution of the Equity Premium Puzzle," Journal of Political Economy, June 1990, 98(3), pp. 519-43.

Epstein, L. G. and S. E. Zin (1989): "Substitution, Risk Aversion, and the Temporal Behavior of Consumption Growth and Asset Returns: A Theoretical Framework," Econometrica, 57(4), 937-69.

- (1991): "Substitution, Risk Aversion, and the Temporal Behavior of Consumption Growth and Asset Returns: An Empirical Analysis," Journal of Political Economy, 99(2), 263-86.

Kihlstrom, R. E. and L. J. Mirman (1974): "Risk aversion with many commodities," Journal of Economic Theory, Volume 8, Issue 3, pp. 361-388

Kreps, D. M., and E. L. Porteus (1978): "Temporal Resolution of Uncertainty and Dynamic Choice Theory," Econometrica, 46, 185-200.

Loewenstein, G. and D. Prelec (1992): "Anomalies in Intertemporal Choice: Evidence and an Interpretation," The Quarterly Journal of Economics, Vol. 107, No. 2, pp. 573-597. 
Mehra, R., and E.C. Prescott (1985): "The Equity Premium: a Puzzle," Journal of Monetary Economy 15:145-161.

Phelps, E. S. (1962): "The Accumulation of Risky Capital: A Sequential Utility Analysis," Econometrica 30 (October 1962): 729-743.

Selden, L. (1978): "A New Representation of Preference over "Certain X Uncertain" Consumption Pairs: The "Ordinal Certainty Equivalent" Hypothesis," Econometrica, 46, 1045-1060.

Starmer, C. (2000): "Developments in Non-Expected Utility Theory: The Hunt for a Descriptive Theory of Choice under Risk," Journal of Economic Literature, Vol. 38, No. 2., 332-382.

Strotz, R. H. (1955): "Myopia and Inconsistency in Dynamic Utility Maximization," Review of Economic Studies, 23 (3), 1955-56. 


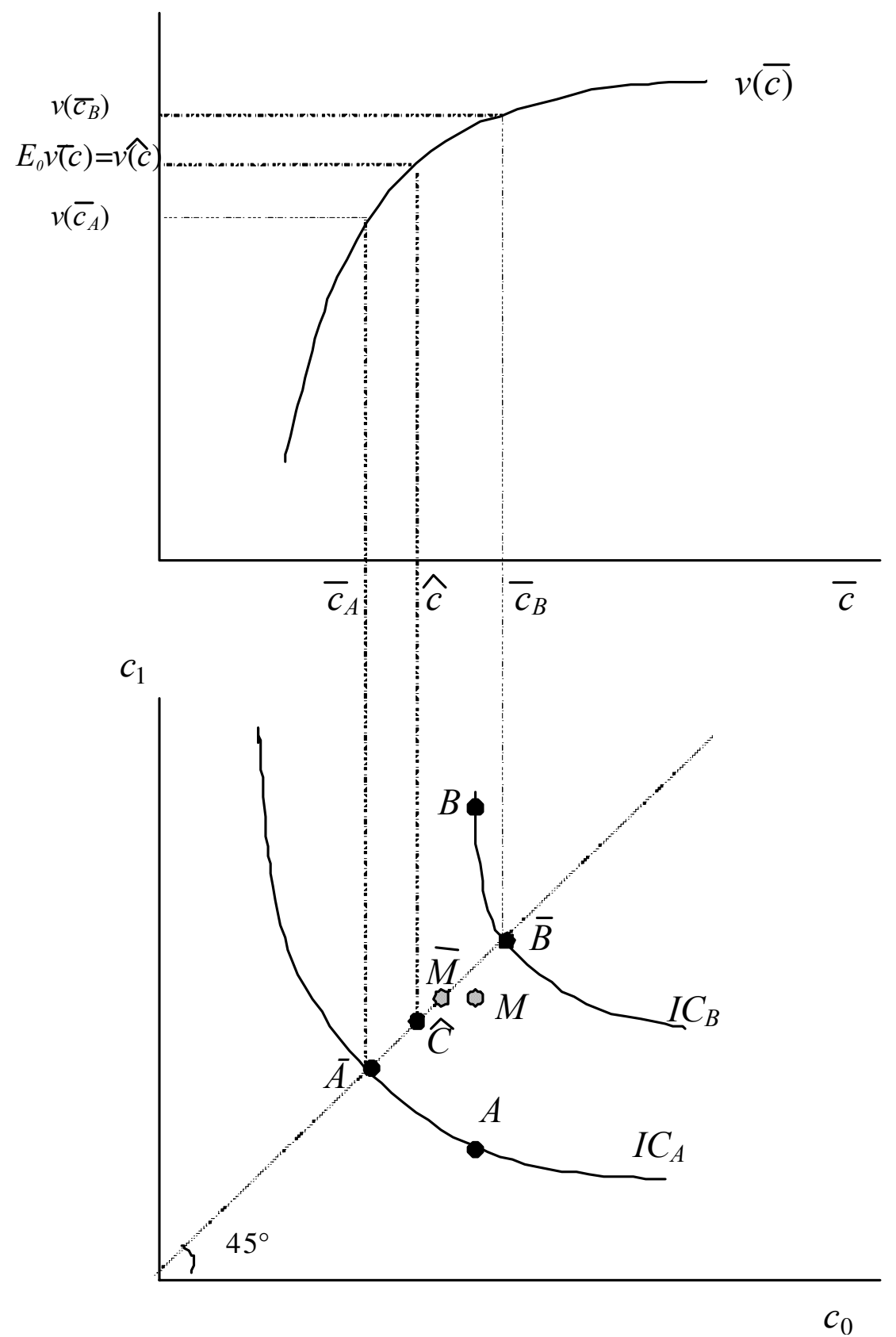

Figure 1: Constancy Equivalence EUT 